\title{
Methods for Three-Dimensional All-Optical Manipulation of Neural Circuits
}

\author{
Emiliano Ronzitti, Valentina Emiliani and Eirini Papagiakoumou*
}

Wavefront Engineering Microscopy Group, Photonics Department, Institut de la Vision, Sorbonne Université, Inserm S968, CNRS UMR7210, Paris, France

Optical means for modulating and monitoring neuronal activity, have provided substantial insights to neurophysiology and toward our understanding of how the brain works. Optogenetic actuators, calcium or voltage imaging probes and other molecular tools, combined with advanced microscopies have allowed an "all-optical" readout and modulation of neural circuits. Completion of this remarkable work is evolving toward a three-dimensional (3D) manipulation of neural ensembles at a high spatiotemporal resolution. Recently, original optical methods have been proposed for both activating and monitoring neurons in a 3D space, mainly through optogenetic compounds. Here, we review these methods and anticipate possible combinations among them.

\section{OPEN ACCESS}

Edited by:

Marco Canepari,

UMR5588 Laboratoire

Interdisciplinaire de Physique (LIPhy),

France

Reviewed by:

Albrecht Stroh,

Johannes Gutenberg University

Mainz, Germany

Marco Lorenzo Dal Maschio, Università degli Studi di Padova, Italy

*Correspondence:

Eirini Papagiakoumou eirini.papagiakoumou@inserm.fr

Received: 04 September 2018 Accepted: 19 November 2018 Published: 17 December 2018

Citation:

Ronzitti E, Emiliani V and Papagiakoumou E (2018) Methods for Three-Dimensional All-Optical Manipulation of Neural Circuits. Front. Cell. Neurosci. 12:469. doi: 10.3389/fncel.2018.00469
Keywords: light-shaping, three-dimensional photostimulation, three-dimensional functional imaging, all-optical neuronal studies, optogenetics, neural circuits

\section{INTRODUCTION}

Controlling and monitoring neuronal activity with a light has become a common practice in many neurobiological studies, throughout the last decade. The continuously expanding toolbox of molecular probes that activate/inhibit (Herlitze and Landmesser, 2007; Airan et al., 2009; Levitz et al., 2013; Klapoetke et al., 2014; Shemesh et al., 2017; Becker et al., 2018; Guruge et al., 2018; Mardinly et al., 2018) or image (Emiliani et al., 2015; Kim et al., 2018) neuronal activity as well as the development of original light-microscopy methods for stimulating these tools (Ronzitti et al., 2017b; Chen et al., 2018c; Yang and Yuste, 2018), has tremendously contributed to the direction of research and has led to innovative experimental concepts (Rickgauer et al., 2014; Carrillo-reid et al., 2016). Photostimulation via optogenetics and/or uncaging (Kwon et al., 2017) is suitable for single cell and, most importantly, circuit studies, since light gives access to a large number of targets simultaneously, at high spatial precision via parallel illumination methods (Papagiakoumou et al., 2010; Packer et al., 2015; Forli et al., 2018; Yang et al., 2018).

Circuit studies are usually performed in vivo by light-stimulation at near-infrared to minimize scattering effects and optimize spatial resolution via non-linear multiphoton absorption processes. Ideally, these studies also demand three-dimensional (3D) accessibility both for activation and imaging at physiological time scales (few-ms scale activation and imaging). 3D imaging approaches enable the use of complementary strategies to access volumes extending up to a few hundred $\mu \mathrm{ms}$ in the axial direction, by proposing the use of piezo scanners to scan the objectives in specific trajectories (Göbel et al., 2007), acousto-optic deflectors (Reddy et al., 2008; Grewe et al., 2010; Katona et al., 2012; Nadella et al., 2016), tunable lenses (Grewe et al., 2011; Fahrbach et al., 2013; Kong et al., 2015), spatiotemporal multiplexing (Cheng et al., 2011; Ducros et al., 2013), light field 
microscopy (Prevedel et al., 2014), or Bessel beam excitation ( $\mathrm{Lu}$ et al., 2017) and reaching tens of $\mathrm{Hz}$ imaging frequencies, of neuronal activity in vivo (Göbel et al., 2007; Grewe et al., 2011; Katona et al., 2012; Nadella et al., 2016). 3D functional imaging of neurons has more recently been demonstrated at even larger volumes, reaching $0.5 \mathrm{~mm}$ in $z$, using a temporally focused Gaussian beam excitation at the size of neuron soma (Prevedel et al., 2016), with large field views up to $5 \mathrm{~mm}$ in $x y$ (Sofroniew et al., 2016; Stirman et al., 2016) or in two different areas of the brain (Lecoq et al., 2014; Chen et al., 2016).

The development of $3 \mathrm{D}$ photoactivation methods is more recent. These systems are based on the use of ComputerGenerated Holography (CGH) (Papagiakoumou et al., 2018; Yang and Yuste, 2018). Although this technique was established for the projection of 3D patterns (Piestun et al., 1996; Haist et al., 1997) or diffraction-limited spots (Liesener et al., 2000) via spatial light modulators (SLMs) several years ago, its use in neuroscience for simultaneous activation of multiple targets in two (Lutz et al., 2008; Nikolenko et al., 2008; Dal Maschio et al., 2010) or three dimensions (Yang et al., 2011; Go et al., 2012; Hernandez et al., 2016; Dal Maschio et al., 2017) was established only during the last decade. Thanks to 3D-CGH, used either solely (parallel methods) or in its diffraction-limit version in combination with scanning of the holographic beamlets (hybrid methods) [see (Papagiakoumou, 2013; Ronzitti et al., 2017b) for detailed description of these approaches], it is possible nowadays to activate multiple neurons providing both the adequate temporal resolution, as well as the spatial resolution for single-cell precision (Figures 1A,B).

With regards to temporal resolution, it is helpful to define the notion of temporal precision in optogenetic activation, i.e., the degree of reproducibility of the occurrence timing of a photo-evoked AP (also indicated as photo-evoked spike jitter) (Figure 1C) and temporal resolution, i.e., the time needed to photo-evoke an AP, ultimately linked to the maximum achievable light-driven neuronal firing rate (Figure 1D). Minimizing those two parameters helps to reproduce precise temporal patterns of activity that in combination with multicell activation, enables to mimic the physiological activity of a network. It has been shown that parallel photoactivation methods (Ronzitti et al., 2017b) can easily achieve short timescales during optogenetic activation (few-ms temporal resolution and sub-ms jitter) (Chaigneau et al., 2016; Ronzitti et al., 2017a; Shemesh et al., 2017; Chen et al., 2018b). Later studies using scanning methods, have also shown a high temporal specificity, reaching a millisecond jitter by using high power in the excitation spots (Yang et al., 2018).

With regards to $3 \mathrm{D}$ spatial resolution, scanning methods using 3D-CGH show an intrinsically good spatial resolution, thanks to the small spot size of the excitation beam (close to diffraction limit). Nevertheless, resolution can dramatically decrease when using intensities close to the saturation levels for the opsin, which results in out-of-focus excitation (Rickgauer and Tank, 2009; Andrasfalvy et al., 2010). Parallel methods use illumination shapes that cover the whole cell body, in order to achieve parallel recruitment of all opsins on the cell membrane to improve efficiency (Papagiakoumou et al., 2010). This, however, causes a quick deterioration of the axial resolution, which scales linearly or quadratically with lateral size, for holographic or Gaussian beams, respectively (Oron et al., 2012). Parallel methods can provide a good axial resolution when combined with temporal focusing (TF) (Papagiakoumou et al., 2008, 2010; Oron et al., 2012; Rickgauer et al., 2014). Notably, temporally focused lightshaping methods allow to preserve sharp borders of the excitation pattern (Papagiakoumou et al., 2010), even through scattering media (Bègue et al., 2013; Papagiakoumou et al., 2013). However, because TF works by dispersing the spectral frequencies of a femtosecond light pulse at a specific plane (Oron et al., 2005), special configurations are needed to extend the methods in $3 \mathrm{D}$.

Here we review the methods proposed so far for $3 \mathrm{D}$ photoactivation and present the possibilities for combination with $3 \mathrm{D}$ imaging modalities, to establish precise and flexible microscopy methods for all-optical manipulation of neural circuits. The methods we present here have mostly been developed for optogenetic photostimulation but in principle, any other photoactivation technique, such as uncaging of caged neurotransmitters, or activation of photoactivable proteins, could benefit from them.

\section{ALL-OPTICAL MANIPULATION}

The very first experiment of all-optical manipulation of neurons was demonstrated by activating cells in neocortical slices via twophoton (2P) uncaging of MNI-glutamate with multiple beamlets generated with a diffractive optical element, or one single beam multiplexed in time, and $2 \mathrm{P} \mathrm{Ca}^{2+}$ imaging (Nikolenko et al., 2007). Similarly, a few years later in two other papers researchers measured $\mathrm{Ca}^{2+}$ signals in neurons while uncaging MNI-glutamate (Dal Maschio et al., 2010; Anselmi et al., 2011). In (Dal Maschio et al., 2010) the optical system incorporated 2P 2D-CGH in the optical path of a commercial 2P scanning microscope and it could exchange holographic or scanning stimulation between the uncaging and imaging beams. In (Anselmi et al., 2011) 3D-CGH with diffraction-limited spots was combined with a remote-focusing system (Botcherby et al., 2007, 2012) to perform functional imaging along tilted dendrites of hippocampal pyramidal neurons in brain slices. Although the first demonstrations of combined activation and imaging of neurons used uncaging, the term all-optical is mostly related to the combination of functional imaging and optogenetic activation. In 2014, a milestone was achieved when an important number of scientific studies showing the activation of neurons in vivo in rodents via optogenetic molecules and the imaging of $\mathrm{Ca}^{2+}$ responses with GCaMP, took place (Vogt, 2015), using either visible (Szabo et al., 2014) or 2P light stimulation (Rickgauer et al., 2014; Packer et al., 2015). More publications followed, using 2D (Carroll et al., 2015; Carrillo-reid et al., 2016; Bovetti et al., 2017; Förster et al., 2017; Forli et al., 2018), and more recently 3D stimulation (Hernandez et al., 2016; Dal Maschio et al., 2017; Mardinly et al., 2018; Yang et al., 2018).

Despite these very important studies, full optical neuronal control remains a challenge in terms of achieving reliable delivery and expression of sensors and actuators in the same neurons, eliminating the cross-talk between imaging and activation, 


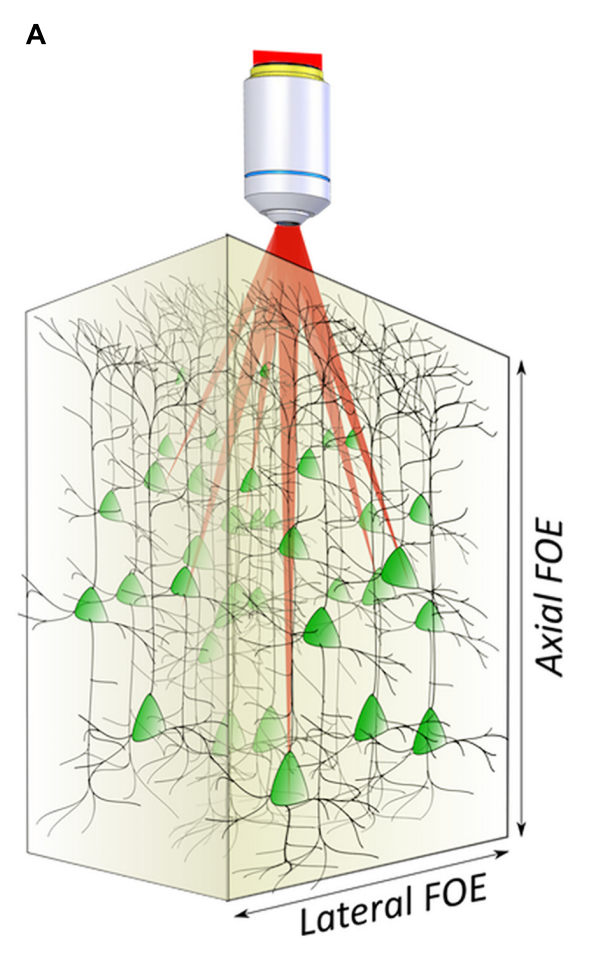

B

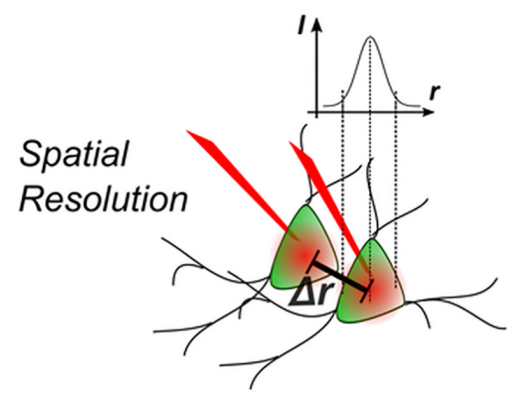

C

Temporal

Precision

D

Temporal

Resolution

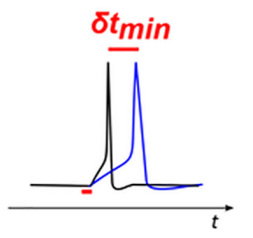

$1 / f_{\text {max }}$

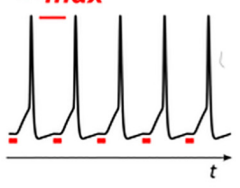

FIGURE 1 | 3D light-targeted photostimulation. (A) SLM-based multiplexing strategies allow to target opsin-expressing neurons over axial and lateral fields of excitation, extending over a few hundred microns in the brain. (B) The photostimulation resolution defines the minimal distance $\Delta r$ between two targets, at which each target can be individually activated. (C) Photostimulation temporal performances are linked to the photostimulation temporal precision, that is the timing precision $\delta t$ in evoking action potentials (APs) with repetitive stimulations (i.e., light-evoked spike jitter) and (D) the temporal resolution, that is the minimal time interval between two consecutive light-evoked APs (i.e., the maximum light-driven neuronal firing rate $f_{\max }$ ).

and recording and stimulating with a single-neuron and a single-action-potential precision (Emiliani et al., 2015). These problems have been discussed exhaustively in other recent reviews (Emiliani et al., 2015; Ronzitti et al., 2017b; Chen et al., 2018c). Here we will focus on reviewing the recent developments for 3D all-optical manipulation.

\section{D Photoactivation}

\section{Fully Parallel Methods}

Fully parallel optical methods proposed for $3 \mathrm{D}$ activation in an all-optical configuration have been presented for optogenetics and make use of extended light-pattern formation to cover the entire neuron soma. Light-patterns can be either large Gaussian beams generated by underfilling the objective numerical aperture (NA; low-NA Gaussian beams), or beams created with more flexible light-patterning methods such as $\mathrm{CGH}$, generalized phase contrast (GPC) or amplitude modulation. As already mentioned, the limit of extended light patterns is the deterioration of the axial confinement, an issue that can be solved by using temporal focusing. Common experimental configurations of TF make use of a diffraction grating in a plane conjugate to the focal plane of the microscope objective (image formation plane), separating the spectral frequencies of the laser femtosecond pulses (dispersion of different wavelengths in different angles) (Oron et al., 2005; Zhu et al., 2005). In other words, the image projected at the grating plane is the image formed at the sample plane, while
$2 \mathrm{P}$ absorption of light projected in any other plane before or after the grating, as it is the case when you generate a $3 \mathrm{D}$ pattern distribution, will be strongly weakened by the pulse broadening. This has, until recently, limited TF-light shaping to $2 \mathrm{D}$ configurations. Methods have been proposed for axial displacement of the TF plane for Gaussian beams, by introducing group velocity dispersion (Durst et al., 2006, 2008; Dana and Shoham, 2012). However, they are only suitable for remotely displacing one plane and they are not compatible with lightpatterning techniques (Leshem et al., 2014), as they can displace the TF plane but not the spatial focusing one.

Hernandez et al. (2016) solved the problem, by introducing the axial displacement mechanism after the grating for TF, which decoupled lateral light shaping from axial displacements. The system used a conventional 2D-CGH with TF for lateral lightpatterning and a second SLM placed at a Fourier plane after the grating to introduce the desired axial shift from the original focal plane, via a lens-effect phase modulation. This configuration also enabled the generation of different excitation patterns at distinct axial planes, by addressing the two SLMs in multiple regions, tiled vertically to the direction of dispersion for TF. With this configuration, researchers demonstrated for the first time the generation of multi-plane temporally focused patterns, reaching a volume of $240 \times 240 \times 260 \mu \mathrm{m}^{3}$ with the axial confinement varying from $5 \mu \mathrm{m}$ Full Width Half Maximum (FWHM) at the center of the field of excitation (FOE) to $10 \mu \mathrm{m}$ at the edges 
of it, tested with spots of $20 \mu \mathrm{m}$ in diameter. The number of regions equal to the number of planes to be addressed. The system was used for selective $2 \mathrm{P} 3 \mathrm{D}$ photoconversion of the Kaede protein (Isobe et al., 2010) in the brain of zebrafish larvae (photoconversion at $800 \mathrm{~nm}$ with $0.1-4.0 \mathrm{~mW} / \mu \mathrm{m}^{2}$ depending the illumination duration) and for $2 \mathrm{D}$ optogenetic activation of ChR2 in zebrafish spinal cord neurons co-expressing GCaMP5G (excitation at $900 \mathrm{~nm}$ with $0.6 \mathrm{~mW} / \mu \mathrm{m}^{2}$ ). Monitoring of $\mathrm{Ca}^{2+}$ traces in that case was performed with visible illumination and two-color HiLo imaging (Lim et al., 2008) (at $~ 30.8 \mu \mathrm{W} / \mathrm{mm}^{2}$ ).

Despite the flexibility of this system, an inherent limitation occurs in the maximum number of axial planes that could be addressed because of the physical tiling of the SLMs, before the quality of the holographic spots get distorted ( $\sim 6$ planes) (Hernandez et al., 2016). Although this can be sufficient for a number of biological applications (Yang et al., 2016), three new studies, have recently proposed ways to increase both the number of planes and the FOE. They all used the same principle: having a beam-shaping method for creating a $2 \mathrm{D}$ temporally focused pattern and using a SLM at a Fourier plane of the TF system for lateral and axial multiplexing of this pattern in several positions in 3Ds via 3D-CGH (Figure 2A). 3D-CGH is used to generate arrays of $3 \mathrm{D}$ diffraction-limited spots, using variations of the Gerchberg-Saxton algorithm (Gerchberg and Saxton, 1972; Di Leonardo et al., 2007) to calculate the phase profile to address to the SLM.

In two of these works the light-shaping part is a TF-Gaussian beam (Pégard et al., 2017; Sun et al., 2018) (Figure 2B). In (Pégard et al., 2017) 3D scanless holographic optogenetics with temporal focusing (3D-SHOT) was used to generate a large number of temporally focused spots, each of them fitting the size of a cell soma of pyramidal neurons (i.e., 10-15 $\mu \mathrm{m}$ FWHM lateral size). With this approach, researchers reported the possibility to project hundreds of excitation spots in a total volume of $350 \times 350 \times 280 \mu^{3}$. (Sun et al., 2018) presented a very similar approach as the one presented by (Pégard et al., 2017), using a TF experimental configuration with two gratings instead of a single grating and a lens. The beam's lateral size was $2.5 \mu \mathrm{m}$ in diameter and the axial FWHM of the 2P light intensity was $7.5 \mu \mathrm{m}$. This study did not include any biological demonstration. The authors used the generated spots in direct laser writing inside a glass, which relies on non-linear light absorption at the focus. Fabrication was conducted either on the surface or inside standard microscope glass slides. As a novelty, they showed that they were able to place focal points at high lateral proximity (4 ţm) with minimal interference between them. That is thanks to the pulse front tilt effect, a property inherent to TF systems where the arrival time of an ultrashort pulse in a certain plane varies across the beam profile thus creating a tilt between the pulse front and the direction perpendicular to the beam. In this way, adjacent spots can be spatially overlapped (Sun et al., 2018).

Both of these studies use Gaussian beams, which is technically simpler compared to the 3D-CGH-TF system proposed by (Hernandez et al., 2016). However, a considerable drawback of this system is the fact that the laser beam is focused on a line on the SLM used for 3D multiplexing, where the size depends on the linear dispersion of the TF system in the direction of dispersing the spectral frequencies (usually parallel to the optical table), and it equals the monochromatic beam size in the unchirped dimension (vertical to the dispersion direction). In the chirped direction, care is usually given to fill the size of the SLM liquid crystal array, while in the unchirped direction the size of the beam is a few millimeters (2-3 mm) (Durst et al., 2008; Sun et al., 2018). This imposes a restriction to the maximum laser power used before damaging the SLM (and thus to the maximum number of spots that can be projected). To overcome this limitation Pégard et al. (2017) proposed adding an extra lens before dispersing the ultrashort pulses on the TF grating, defocusing the beam on the SLM and thus increasing the illumination area. Nevertheless, the defocusing created a secondary spatial focus in the form of a line that deteriorated the axial propagation of the beam (Pégard et al., 2017; Chen et al., 2018c). In a more advanced version of this method the lens was replaced by a rotating diffuser at an image plane, after the grating. This led to an enlarged illumination of the SLM, without secondary focus effects (Mardinly et al., 2018) and Gaussian beams of $23 \mu \mathrm{m}$ axial FWHM (axial optical Point Spread Function; PSF) (Pégard et al., 2018). 3D-SHOT was used in this case to simultaneously stimulate neurons co-expressing newly developed excitatory or inhibitory somatic opsins, ST-ChroME or IRES-ST-eGTACR1, respectively, and GCaMP6s in an all-optical configuration (about 50 neurons in three different planes extending to an axial range of $100 \mu \mathrm{m}, 0.13 \mathrm{~mW} / \mu \mathrm{m}^{2}$ or $40 \mathrm{~mW}$ per target for activating ST-ChroME neurons, $0.08 \mathrm{~mW} / \mu \mathrm{m}^{2}$ or $\sim 6 \mathrm{~mW}$ per target for IRES-ST-eGTACR1 neurons, illumination with a low-repetition rate laser at $1040 \mathrm{~nm}$ for $1 \mathrm{~s}$ ) (Mardinly et al., 2018).

Higher flexibility and better axial resolution was demonstrated by Accanto et al. who presented a system for multiplexed temporally focused-light shaping (MTF-light shaping), where the beam-shaping part was either 2D-CGH or GPC (Accanto et al., 2018) (Figure 2C,D). For MTF-CGH, the optical setup is the same as the one of 3D-CGH-TF (Hernandez et al., 2016), the only difference being the way the multiplexing SLM is addressed. MTF-CGH enabled investigators to generate $15-\mu \mathrm{m}$ diameter temporally focused holographic spots on 50 independent planes on an excitation field of $300 \times 300 \times 500 \mu \mathrm{m}^{3}$ and an average axial PSF of $11 \mu \mathrm{m}$ FWHM. The theoretical FOE for the optical parameters they used was $750 \times 750 \times 990{\mu \mathrm{m}^{3}}^{3}$, but was experimentally limited by the size of the optics used.

In MTF-GPC the beam-shaping part was substituted by a GPC setup (Glückstad, 1996; Papagiakoumou et al., 2010) for projection of high-precision, speckle-less, temporally focused arbitrarily shaped patterns. Notably, the combination of GPC with $\mathrm{CGH}$ to extend GPC to 3D was previously reported (Go et al., 2011; Bañas and Glückstad, 2017) but without TF, which is essential for suppressing excitation by the out-of-focus light (Papagiakoumou et al., 2010). In MTF-GPC, characterization of $12-\mu \mathrm{m}$ diameter TF-GPC spots showed improved axial PSF, compared to MTF-CGH (6 $\mu \mathrm{m}$ FWHM on average on a FOE of $200 \times 200 \times 200 \mu^{3}$ ), as expected for TF-GPC patterns (Papagiakoumou et al., 2010). Similar to the Gaussian beam case, a crucial drawback remains the illumination of the multiplexing SLM with a line. To overcome this, (Accanto et al., 2018) removed the phase contrast filter and used the first SLM of their 
A

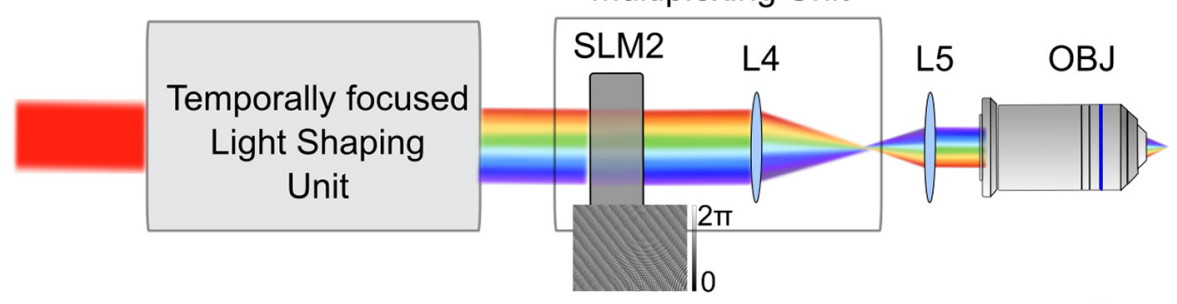

B

TF - Light Shaping Unit

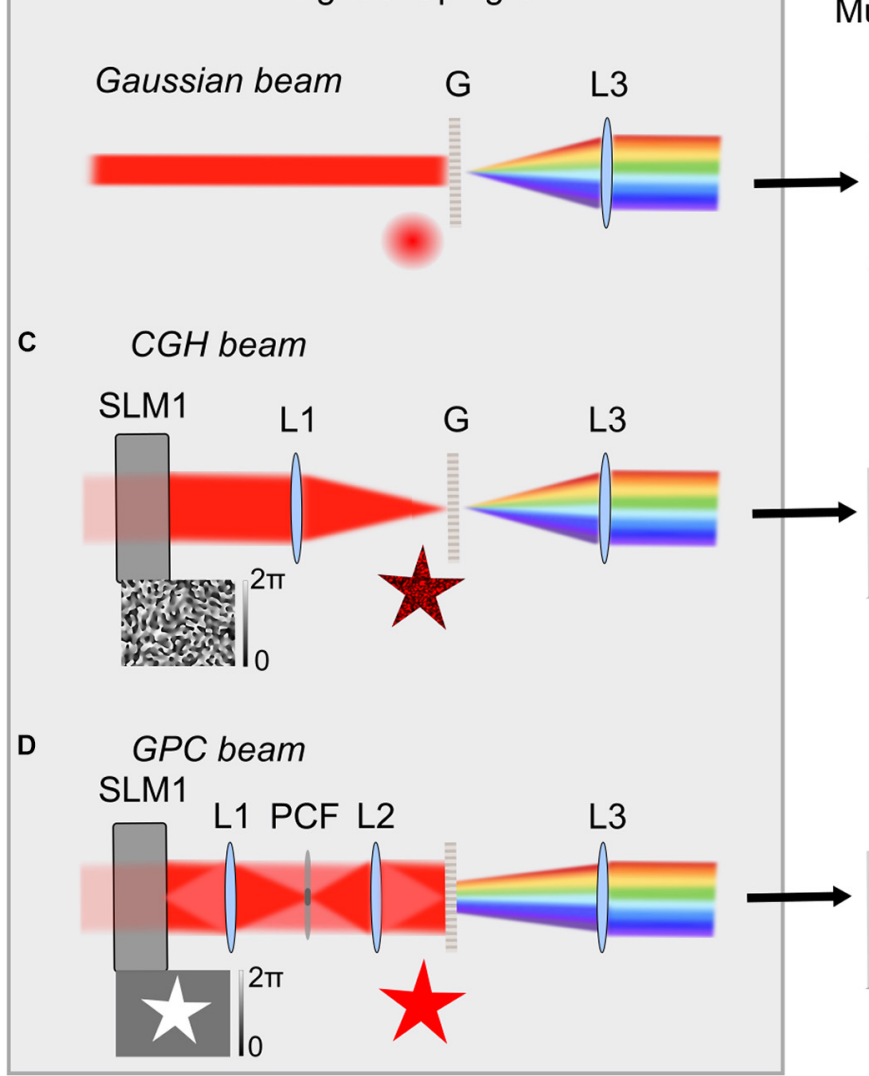

Multiplexed Pattern

Experimental Demonstration
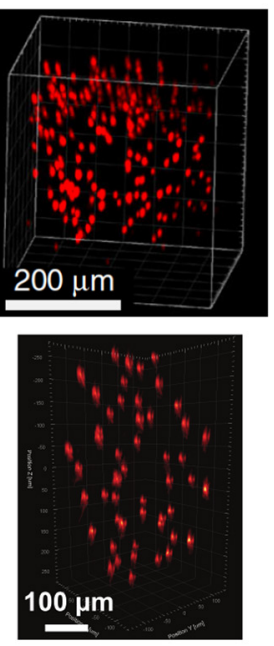

FIGURE 2 | 3D multiplexed temporally focused light shaping. (A) Optical systems for 3D temporally focused light shaping consist of a light-shaping unit for creating a 2D temporally focused pattern and a multiplexing unit, using a SLM (SLM2) at a Fourier plane of the TF system, to replicate the 2D pattern in several positions in 3D via 3D-CGH (example of a phase profile for projecting 3 diffraction-limited spots in different positions is shown on the bottom of SLM2). After the light-shaping unit, the beam is represented with its spectral frequencies diffracted, because of spectral diffraction by the grating G. After phase modulation on SLM2 the beam is imaged by two lenses (here L4 and L5) to the back aperture of the microscope objective. (B-D) Different cases for the TF-light-shaping unit: (B) Gaussian beam. In this case the grating is illuminated with a collimated Gaussian beam of a suitable size, usually adjusted with a telescope of lenses (not shown here). Configuration with a non-collimated beam (by introducing a lens prior to the grating) was used by Pégard et al. (2017) in order to increase the illuminated area of SLM2. Middle. Illustration of the example for projection of 3 Gaussian replicas, when the SLM2 is illuminated with the phase profile shown in A. Right. Experimental demonstration showing projection of 200 Gaussian beams in a $350 \times 350 \times 280 \mu \mathrm{m}^{3}$ volume, adapted from Pégard et al. (2017). (C) CGH beam. A SLM (SLM1) and a lens (L1) are used for holographic pattern projection (here, a star), that is then replicated in the 3 different positions (Middle). Right. Experimental demonstration of 50 holographic circular spots of $15 \mu \mathrm{m}$ diameter in a $300 \times 300 \times 500 \mu \mathrm{m}^{3}$ volume, adapted from Accanto et al. (2018). (D) GPC beam. SLM1 is used for a binary phase modulation of $\Delta \varphi=\pi$, that is then phase contrasted by the phase contrast filter (PCF), placed at the focal plane of $L 1$. A sharp speckle-free pattern is formed at the grating plane by L2. Middle. 3 replicas for the GPC pattern in the corresponding predefined positions of the multiplexing unit. Right. Experimental demonstration of projection of 17, 12- $\mu \mathrm{m}$ diameter circular GPC spots in a $200 \times 200 \times 200 \mu \mathrm{m}^{3}$ volume, adapted from Accanto et al. (2018). L3 Collimating lens.

configuration to perform amplitude and phase modulation. They encoded a pattern in four different areas on the beam-shaping SLM and the pattern of each area was laterally displaced with a different prism-phase effect, such as generating four different lines on the multiplexing SLM after the beams were temporally focused. Addressing the first SLM in different areas enabled the projection of replicas of four different speckle-free patterns in a volume of $300 \times 300 \times 400 \mu^{3}$, a method referred to as MTF-Multi Shapes. This strategy both increased the illumination area of the multiplexing SLM and allowed more flexibility on 


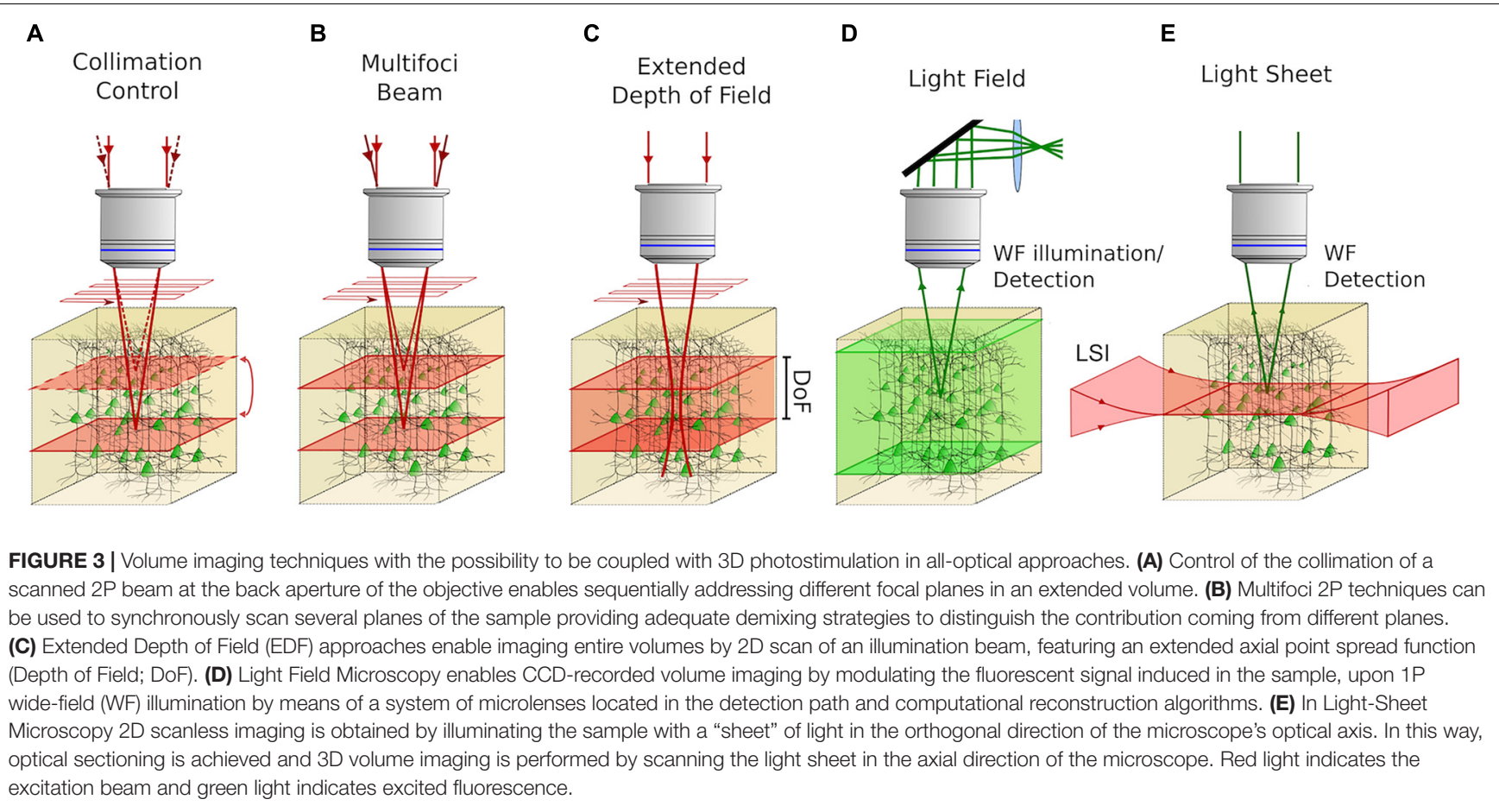

the shape of the projected patterns, similar to that described by (Hernandez et al., 2016).

Evidently, flexibility of the MTF-light shaping methods comes at the cost of simplicity of the optical setup and total cost. For simpler cost-effective solutions in applications where the excitation spot size and form can be predetermined, it is possible to use static lithographically fabricated phase masks (Accanto et al., 2018) to replace the first SLM in MTF-CGH, or a GPC-light shaper (Bañas et al., 2014) to replace the GPC setup in MTF-GPC.

MTF-CGH was used in a multi-cell excitation of photoactivatable GCaMP (paGCaMP) in the central nervous system of the drosophila larvae (photoconversion at $760 \mathrm{~nm}$ with $1.0 \mathrm{~mW} / \mu \mathrm{m}^{2}$, illumination with trains of $10-\mathrm{ms}$ pulses at $50 \mathrm{~Hz}$, total illumination duration: tens of seconds up to $4 \mathrm{~min}$ ) and to photoconvert Kaede in the zebrafish larva hindbrain (photoconversion at $800 \mathrm{~nm}$ with $0.4 \mathrm{~mW} / \mu \mathrm{m}^{2}$, illumination with trains of $10-\mathrm{ms}$ pulses at $50 \mathrm{~Hz}$, total illumination duration 1-4 min). Parallel illumination of neurons allowed fast photoconversion in both cases, with minimal photoactivation of untargeted neighboring cells. Especially in the case of paGCaMP, neuronal processes of the targeted cells could be clearly distinguished from the background, allowing the possibility to precisely track neuronal morphology (Accanto et al., 2018).

Although the use of TF in neuronal photoactivation with parallel methods has offered the possibility to locally confine the excitation volume, such as to preserve single-cell resolution, this might not be necessary for low-scattering samples, excitation of small size cells or sparse staining. In that case, 3D-CGH alone can be used for the projection of extended light patterns in different planes (Haist et al., 1997; Hernandez et al., 2016). Thus 2P 3D-CGH spots of $6-\mu \mathrm{m}$ diameter were used to photoactivate ChR2 in the zebrafish larval neurons, which in combination with 2P GCaMP6s $\mathrm{Ca}^{2+}$ imaging, enabled the identification of neuronal ensembles associated with control of tail bending (photoactivation at $920 \mathrm{~nm}, 0.2 \mathrm{~mW} / \mu \mathrm{m}^{2}$ or 50 $\mathrm{mW}$ per target, circular overlapping photoactivation regions of $18 \mu \mathrm{m}$ in diameter) (Dal Maschio et al., 2017). Moreover, the authors performed targeted photoactivatable GFP (paGFP) photoconversion to obtain a morphological reconstruction of individual functionally identified neurons (photoconversion at $750 \mathrm{~nm}$ with $0.25 \mathrm{~mW} / \mu \mathrm{m}^{2}$ or $7 \mathrm{~mW}$ per target, 1-s illumination).

Finally, as a general comment we should note that $3 \mathrm{D}$ TF methods using CGH for multiplexing the excitation spot, can produce powerful experimental configurations in volumetric FOEs, with the quality of all spots being the same across the whole FOE, since the spots multiplexed are replicas of the same single original spot. However, for volumes reaching mm-range, care should be taken to homogenize the excitation properties of the projected spots, by taking into account factors such as scattering with increasing depth, SLM-diffraction-efficiency corrections, optical aberrations due to the large defocusing of spots (objective used at its limits) or projection near the borders of the FOE, and spectral aberrations for TF occurring by cropping spectral frequencies in the optics when using large defocus (Hernandez et al., 2016).

\section{Hybrid Methods}

Scanning methods are alternative approaches for neuronal stimulation and have been widely used in $2 \mathrm{P}$ optogenetics (Rickgauer and Tank, 2009; Andrasfalvy et al., 2010; Packer et al., 2012; Prakash et al., 2012), mainly for the activation of the slow kinetics opsin, C1V1, and most of the times in a spiral trajectory (Packer et al., 2012, 2015; Carrillo-reid et al., 
2016; Yang et al., 2018). They represent the simplest and most immediate solution for many laboratories, since they adopt conventional $2 \mathrm{P}$ scanning microscopes based on galvanometric scanners. Nevertheless, the sequential photostimulation limits the achievable temporal resolution (Papagiakoumou, 2013; Ronzitti et al., 2017b) and does not allow the simultaneous activation of multiple targets. The use of resonant scanners or acousto-optic deflectors (AODs) to increase the temporal resolution of scanning methods, is still limited by the necessary dwell time in 2P excitation, especially for slow opsins (Prakash et al., 2012). Moreover, due to their cycling at resonant frequencies $(8 \mathrm{kHz})$, resonant scanners cannot provide the necessary flexibility for arbitrary excitation trajectories, like spiral scans. For simultaneous multicell activation in 3Ds, scanning microscopes can be modified to include a SLM to multiplex the beam prior to the scan via 3D-CGH (Packer et al., 2012; Yang et al., 2018). The holographic pattern, consisting of multiple neardiffraction limited spots ( $\sim 1 \mu \mathrm{m}$ in diameter), is then scanned in spiral trajectories on an area covering the size of the cell soma (Rickgauer and Tank, 2009; Packer et al., 2015; Yang et al., 2018).

Studies using hybrid methods in all-optical configurations have so far used C1V1 as optogenetic actuator excited at $1040 \mathrm{~nm}$ (Packer et al., 2015; 20-80 $\mathrm{mW}$ per target, spirals of $20 \mathrm{~ms}$, $80 \mathrm{MHz}$ repetition rate laser; Yang et al., 2018; 2.2-6.0 $\mathrm{mW}$ per target, spirals of $20 \mathrm{~ms}$, low-repetition rate amplified laser). For $3 \mathrm{D}$ manipulation of neurons specifically, there is currently only one study where investigators simultaneously photostimulated more than 80 neurons over $150 \mu \mathrm{m}$ in depth in layer $2 / 3$ of the mouse visual cortex, while simultaneously imaging the activity of the surrounding neurons with GCaMP6s (Yang et al., 2018). The authors photoactivated in three different planes in an axial range of $100 \mu \mathrm{m}$ selected groups of somatostatin inhibitory interneurons, suppressing the response of nearby pyramidal neurons to visual stimuli in awake animals $(6 \mathrm{~mW}$ per cell from a low-repetition rate laser, or $6 \mathrm{~mW} / \mu^{2}$, since the surface of the illumination spot in that case is about $1 \mu \mathrm{m}^{2}$, illumination for $2.8 \mathrm{~s}$ with 175 continuously repeated spirals, each lasting $\sim 16 \mathrm{~ms}$ ).

\section{Maximum Number of Excitation Targets}

An estimate of the maximum possible number of targets to address with each approach in the framework of an all-optical experiment, presumes knowledge of the total light losses of an optical system from the laser source to the objective output, which can significantly vary from one system to another. Moreover, the power necessary per target used, can vary according to opsin type, expression level, cell health and activation depth. In general, power losses for parallel illumination methods mainly consist of losses on the $\operatorname{SLM}(\mathrm{s})$ and the diffraction grating used for TF. For hybrid methods losses are approximately 23 times less than those of parallel ones, since they are mainly due to the use of a single SLM. It has also been reported that parallel approaches need about twice the power used by a spiral scanned beam, to induce a neuronal response with the same properties in both cases (Picot et al., 2018; Yang et al., 2018). Thus, in principle, for the same laser source and systems carefully designed to minimize power losses, hybrid methods are supposed to outmatch parallel ones about 4 times in the maximum number of cells possibly activated (without considering photodamage limits). From what is reported so far in literature, the maximum number of cells that have been simultaneously activated with parallel approaches is 50 neurons (Mardinly et al., 2018), while for hybrid methods this number is reported to be up to 80 (Yang et al., 2018). In the first case, the authors clearly state that they were limited by the available power of their laser system. However, current advances in laser technology can provide fiber amplified systems that deliver up to $60 \mathrm{~W}$ maximum average power, allowing for the possibility to greatly increase the above reported numbers.

A fundamental difference between parallel and hybrid 3D multi-target photoactivation methods is the eventual photodamage effects that one can induce by increasing the number of targets and thus the amount of light that is sent to the tissue. As presented in the previously reported cases, in general, parallel illumination approaches use lower illumination intensity $\left[<0.4 \mathrm{~mW} / \mu \mathrm{m}^{2}\right.$ independently on the opsin type, see also (Chen et al., 2018b)] but higher total average power per target (e.g., for $2 \mathrm{P}$ in vivo activation $10-45 \mathrm{~mW}$ when amplified laser pulses of tens of $\mu \mathrm{J}$ pulse energy are used from lowrepetition rate fiber amplifiers, 30-90 $\mathrm{mW}$ when $\mathrm{nJ}$-energy pulses are used from $\mathrm{MHz}$-repetition rate oscillators) than scanning approaches (Chen et al., 2018b; Forli et al., 2018; Mardinly et al., 2018), making them more vulnerable to heating, i.e., linear photodamage. On the other hand, scanning (or hybrid) methods use high intensity $\left(2-6 \mathrm{~mW} / \mu \mathrm{m}^{2}\right)$ focused beams of low total average power (Yang et al., 2018) (although average powers in the range of $20-80 \mathrm{~mW}$ were reported (Packer et al., 2015) when nJ-energy pulses were used from $\mathrm{MHz}$ repetition rate oscillators), making them vulnerable to non-linear photodamage.

For non-linear photodamage, the damage threshold was shown to be inversely proportional to the pulse duration and proportional to the square of the mean power (König et al., 1999). It has been evaluated on the basis of morphological damage for CHO (Chinese Hamster Ovarian) cells to $0.1 \mathrm{~J} / \mathrm{cm}^{2}$ (König et al., 1999), or tissue ablation for porcine cornea (Olivié et al., 2008) to $1.5-2.2 \mathrm{~J} / \mathrm{cm}^{2}$ for $800-1000 \mathrm{~nm}$. For comparison, an intensity of $80 \mathrm{~mW} / \mu \mathrm{m}^{2}$ at $80 \mathrm{MHz}$ pulse repetition rate corresponds to a fluence of $0.1 \mathrm{~J} / \mathrm{cm}^{2}$. No relevant studies exist for the mouse brain. A recent study on tissue heating, took the standard illumination parameters for either parallel or scanning methods into consideration, showing that the local temperature rise on a target area did not exceed the physiological limits in both cases $(<1 \mathrm{~K})$ (Picot et al., 2018). Specifically, to generate an action potential in vivo, with a holographic spot of $12-\mu \mathrm{m}$ diameter at a depth of $\sim 150 \mu \mathrm{m}$, illuminating a neuron for $3 \mathrm{~ms}$, at $1030 \mathrm{~nm}$ and $0.1 \mathrm{~mW} / \mu \mathrm{m}^{2}$, the average temperature rise over the spot's surface, is estimated to $0.3 \mathrm{~K}$. Furthermore, comparing the temperature rise for experimental conditions able to generate action potentials with latency in the range of $2-10 \mathrm{~ms}$ in vitro, it was found that for a holographic spot (3-ms illumination, $0.2 \mathrm{~mW} / \mu \mathrm{m}^{2}$ ) the average rise was $1 \mathrm{~K}$, while for a focused beam in a spiral trajectory ( $3-\mathrm{ms}$ illumination, $31 \mathrm{~mW} / \mu \mathrm{m}^{2}$ ) the mean temperature rise was $<0.5 \mathrm{~K}$, and the local rise at the center 
of the spiral was again $\sim 1 \mathrm{~K}$ (Picot et al., 2018). For multitarget excitation, what remains critical is the distance between the different targets: for spots placed at an average distance from their nearest neighbor greater than the thermal diffusion length in tissue $\ell_{\mathrm{th}}=\sqrt{6 D t}$, where $D$ is the thermal diffusion constant [140 $\mathrm{\mu m}^{2} / \mathrm{ms}$ (Yizhar et al., 2011), and $t$ the evolved time], the temperature rise remains comparable to the case of the isolated spot (for 3-ms illumination duration, holographic stimulation at an intensity $\leq 0.2 \mathrm{~mW} / \mu \mathrm{m}^{2}$ enables keeping the induced temperature rise $<2 \mathrm{~K}$ for activating 100 cells whose inter-soma distance was larger than the thermal diffusion length, which in that case was $\sim 50 \mu \mathrm{m})$. Otherwise, the heat load starts to significantly increase locally (Picot et al., 2018). Moreover, in terms of illumination duration, prolonged stimulation ( $>1 \mathrm{~min}$ ) was found to induce substantial brain heating $\left(6-8^{\circ} \mathrm{C}\right)$ (Mardinly et al., 2018).

Notably, the considerations of the study by Picot et al. (2018) indicate that the optimal laser repetition rate for $2 \mathrm{P}$ optogenetics depends on the adopted illumination method: the very low excitation intensity used in parallel illumination, allows to neglect non-linear damage effects and privileges using low $(500 \mathrm{kHz}-$ $2 \mathrm{MHz}$ ) repetition rate lasers to minimize heating through linear absorption. Scanning approaches on the other hand, require high excitation intensity but enable more efficient heat dissipation because of their small beam spot size; therefore, for short illumination times, a higher repetition rate laser (Ji et al., 2008) should be preferred in order to minimize peak-power-sensitive damages.

\section{D Imaging}

In order to combine photostimulation and functional imaging over large neuronal populations in extended volumes, it is necessary to elaborate strategies to decouple and independently control the photostimulation and the imaging plane. An exhaustive presentation of the existing imaging techniques have recently been presented in literature (Ji et al., 2016; Yang and Yuste, 2017). Here we will discuss the techniques that can be combined with $3 \mathrm{D}$ photoactivation, in volumetric all-optical studies.

Adoption of approaches for 3D imaging, involving fast mechanical axial movements of the objective lens with piezoelectric positioners (Grewe et al., 2010; Katona et al., 2011), in combination with 3D photoactivation methods can be rather challenging for an independent control of the stimulation and imaging planes. In that case, the microscope objective is shared by both photostimulation and imaging paths, and a 3D alloptical configuration would require a simultaneous readjustment of the axial position of the photostimulation foci, to compensate for the objective shifts. Since 3D photoactivation methods use a SLM to project the excitation spots/patterns to different axial planes at a maximum refresh rate of $3 \mathrm{~ms}$ (Yang et al., 2016), a combination of fast piezo-repositioning approaches is feasible in cases where imaging is done in few discrete axial planes (Cossell et al., 2015; Peron et al., 2015; Seelig and Jayaraman, 2015), but not possible when objectives need to be moved over an extended volume in a quasi-continuous way (Grewe et al., 2010; Katona et al., 2011).

Strategies involving the fast repositioning of the imaging focus by modulating the imaging beam divergence (Figure 3A), appear to be more convenient for all-optical volume investigations. This can be done by introducing a lens-effect in an upstream location of the imaging path, possibly in a plane conjugated to the objective back aperture to obtain a telecentric system. Clearly, the control of laser divergence must be fast enough to be compatible with functional imaging rates. Few technologies are commercially available for high-speed focus control through lenses. They are based either on the curvature change of a flexible-membrane electrically controlled lens (usually referred as Electrically Tunable Lens; ETL) (Grewe et al., 2011) or on ultrasounds propagating in a confined fluid, resulting in a tunable index of refraction gradient lens [usually referred as Tunable Acoustic Gradient (TAG) lens] that behaves like an aspheric lens (Mermillod-Blondin et al., 2008). ETLs have been mainly driven in stepping mode, enabling a $\approx 15 \mathrm{~ms}$ refocus time and can be electronically synchronized with the two-photon scanning imaging acquisition (Grewe et al., 2011). They have been successfully applied in 3D all-optical experiments, enabling simultaneous two-photon imaging and photostimulation on three planes axially spanning over $100 \mu \mathrm{m}$ in mammals (Yang et al., 2018) and on five planes axially spanning over $32 \mu \mathrm{m}$ in zebrafish (Dal Maschio et al., 2017). In TAG varifocal lenses, the optical power varies continuously at resonant frequencies, thus enabling much higher speeds with a $\approx 1 \mu$ s switching time, but they require careful control of oscillations through optical-phase locking (Kong et al., 2015). Volume imaging is built up by stacks of $x z$ planes (where the fast-axis is along the axial direction) resulting in high-rate volume imaging ranging between $14 \mathrm{~Hz}$ $\left(375 \times 112 \times 130 \mu \mathrm{m}^{3}\right)$ and $56 \mathrm{~Hz}\left(60 \times 4 \times 30 \mu \mathrm{m}^{3}\right)$.

Alternatively, SLMs or AODs can be used to dynamically control the degree of divergence of the imaging beam. SLM permits wavefront modulation resulting in fast beam refocusing (Dal Maschio et al., 2011) with refreshing rates up to $300 \mathrm{~Hz}$. Importantly, in this case modulation is not limited to beam refocusing but can potentially be combined with more complex wavefront modulations, to correct optical aberrations (Booth, 2014) or to optimize the signal to noise ratio by targeting illumination of the cell (in this latter case in a CCD detection scheme) (Foust et al., 2015; Bovetti et al., 2017; Tanese et al., 2017). In the latter $2 \mathrm{P}$ near diffraction-limited stationary laser spots generated through $\mathrm{CGH}$ were used to perform scanless high-speed imaging of GCaMP6 activity in neurons in vivo on a CCD camera (Bovetti et al., 2017), or voltage imaging on dendritic spines (Tanese et al., 2017). CGH-shaped excitation was also used to improve the signal to noise ratio in voltage imaging experiments of dendrites and axons (Foust et al., 2015).

AODs can also be used for fast 3D beam repositioning. Using two pairs of orthogonal AODs addressed with counterpropagating acoustic waves of linearly varying frequency (chirped waves), it is possible to impress a precise $x y$ radial deflection (determined by the center frequencies of the waves) and $z$ axial displacement (determined by the amount of chirp) to the 
illumination beam. Being completely inertia-free, AOD systems can achieve very short commutation $(24.5 \mu \mathrm{s}$ for $3 \mathrm{D}$ random access) and dwell (0.05-1.0 $\mu \mathrm{s})$ times (Kirkby et al., 2010; Nadella et al., 2016). This makes AODs especially well-suited for high-rate random access point- or line-scanning imaging. For instance, in (Katona et al., 2012) the authors recorded responses from a population of individual neurons and glial cells, in the visual cortex of adult anesthetized mice, by automated tissuedrift compensation performed plane by plane, when obtaining a reference $\mathrm{z}$-stack or between $3 \mathrm{D}$ random-access scans. They recorded spontaneous activity within $400 \times 400 \times 500 \mu \mathrm{m}^{3}$ at a frequency up to $56 \mathrm{~Hz}$. In another example (Nadella et al., 2016), researchers performed random-access patch imaging of neurons in layer $2 / 3$ of primary visual cortex in an awake behaving mouse at $50 \mathrm{~Hz}$, as well as the simultaneous dendritic and somatic imaging of pyramidal neurons in the visual cortex of awake mice at $27.9 \mathrm{~Hz}$, by applying post hoc movement correction of images. The downside of such systems is that the combination of four AODs in a series is associated with power losses up to 75\% (Reddy et al., 2008; Nadella et al., 2016) and requires strategies to compensate the temporal and spatial dispersion (Katona et al., 2012). However, recent developments in the AOD technology allow more efficient and uniform light transmission over larger scan angles when focusing away from the nominal focal plane of the objective (Nadella et al., 2016).

Remote focusing is another approach enabling fast sequential imaging of axially separated planes. It allows remote axial shifting the imaging beam by integrating a classical raster scanning system with an axial scan unit, which comprises of an objective lens and a lightweight mirror (Botcherby et al., 2007, 2012). Since the mirror is imaged on the sample plane, its oscillations are translated in a rapid change of focus in the sample, without physically moving the imaging objective lens. Fast oscillations are enabled by using a custom-built actuator constructed with a pair of galvanometer motors to scan the mirror in the $\mathrm{kHz}$ range. Importantly, as the two objectives are disposed to modify the beam wavefront with equal and opposite aberrations, the microscope is resilient to the systematic aberrations introduced by diverging beams that yield large focus shifts. Remote focusing enabled volume imaging of extracellular electrically induced calcium transients in OGB-loaded neurons up to $1 \mathrm{kHz}$, over a depth of $60 \mu \mathrm{m}$ (Botcherby et al., 2012). Of note, other systems using remote focusing units, have used voice coil motors to drive the movable mirror at high speed (Rupprecht et al., 2016; Sofroniew et al., 2016).

Other volume imaging approaches, potentially compatible with $3 \mathrm{D}$ photostimulation, are based on multi-foci beams sent in parallel on the sample, imaging different planes simultaneously (Figure 3B), provided that ad hoc read-out demultiplexing strategies are adopted, to distinguish the signal coming from those planes. One possible strategy involves temporally multiplexed beams. Pulsed beams can be sent at different foci with different time delays. If the delays are superior to the fluorescence lifetime decay (in the range of few ns) and inferior to the inter-pulse interval, the fluorescence signal originating from different locations, can be distinguished by temporally demultiplexing the detected signal (Amir et al., 2007;
Cheng et al., 2011). Other strategies rely on computational demultiplexing algorithms, which permit simultaneous multifoci imaging, without introducing any temporal shifts among the beams. In this case, a priori knowledge of the cells distribution and sparsity of cortical neuronal activity, allow demixing signals from different planes, using independent component analysis or non-negative matrix factorization algorithms (Yang et al., 2016).

Further strategies use extended depth of field (EDF) imaging, where the illumination PSF elongates axially (Botcherby et al., 2006). Since several layers of cells are encompassed within the PSF (Figure 3C), a lateral scan of such a beam is equivalent to projecting a stack of axially displaced layers in a single plane. Volume imaging is thus enabled at speeds equal to scan-based planar imaging. Very high-volume rates of functional imaging can thus be obtained, provided that neural activity comes from the sparse distribution of neurons, that do not significantly overlap axially and an a priori high-resolution mapping of the cells position in the volume, is acquired. Bessel beam based EDF functional volume imaging has been reported in vivo at $30 \mathrm{~Hz}$ for volumes extending up to $160 \mu \mathrm{m}$ (Lu et al., 2017).

EDF imaging can also be obtained by engineering the detection PSF. Here, the strategy is conceptually reversed compared to previous approaches: instead of attaining volume imaging by modifying the illumination beam, axial discrimination is achieved by modulating the detected fluorescent signal. Similar to the excitation PSF, the detection PSF can be phase-only modulated with a transparent static phase mask placed at a Fourier plane of the detection path, so that it does not disturb the numerical aperture and the photon throughput of the system (no photon losses). Imaging can then be performed with a CCD and computational tools can be used to recover image information over the entire extended depth of field, as in the case of the elongated excitation PSF. Researchers have shown that the use of cubic phase masks in such configurations, in combination with CGH-based target illumination, allows for simultaneous imaging of fluorescence signals arising from different 3D targeted points (Quirin et al., 2013). Moreover, an a priori information of the origin of the fluorescence signal, through targeted excitation with CGH spots, can remove any ambiguity arising from imaging unknown objects with extended axial features (Quirin et al., 2013). Here, the strategy is conceptually reversed compared to previous approaches: instead of attaining volume imaging by modifying the illumination beam, axial discrimination is achieved by modulating the detected fluorescent signal.

Alternatively, volumetric imaging can be obtained in a wide-field illumination configuration using Light Field Microscopy (LFM) (Broxton et al., 2013) (Figure 3D). A series of micro lenses are placed at the native image plane (i.e., the plane where a camera is put in standard wide-field configurations) and a relay lens system is used to reimage the lenslets' back focal plane onto a camera (Broxton et al., 2013). Since in-focus and out-of-focus light results in different patterns at the camera, axial localization of the emitters in a sample volume can be obtained by computationally processing the image. The volumetric imaging speed 
is only limited by the CCD acquisition rate. Despite the high temporal performances, the application of LFM has been restricted to semi-transparent tissues due to scattering limitations (Broxton et al., 2013; Cohen et al., 2014; Prevedel et al., 2014). However, it has recently been proposed in a computational imaging approach, where it is integrated with high-dimensional, structured statistics enabling fast volumetric acquisition in vivo in the brains of mammals (Grosenick et al., 2017). If coupled with $3 \mathrm{D}$ photoactivation, particular attention needs to be paid when considering photoactivation cross-talk, as contamination induced by a single-photon wide-field imaging beam, may not be negligible.

Finally, light-sheet microscopy (LSM) represents another volumetric imaging approach (Figure 3E) particularly suited to whole-brain imaging of small-scale organisms (Keller and Ahrens, 2015; Power and Huisken, 2017), at single-cell imaging resolution (Ahrens et al., 2013). In this case, optical sectioning of the specimen is obtained in a conventional wide-field detection scheme, with an orthogonal illumination of the sample by means of a thin "sheet" of light coming from the side. Since excitation only yields in an axially confined planar portion of the sample, optically sectioned video-rate imaging of specific planes is enabled simply by using a common camera-based detection. $3 \mathrm{D}$ photostimulation coupling could be, e.g., envisaged by delivering photostimulation light through the high-NA detection objective. All-optical 3D functional investigations might then be obtained by adopting those LSM volumetric strategies involving axial lightsheet repositioning and varifocal ETL-based detection (Fahrbach et al., 2013) or cubic phase-based extended depth of field detection approaches, combined with imaging deconvolution (Olarte et al., 2015; Quirin et al., 2016). It is worth mentioning that, since the side-on light-sheet needs to uniformly excite a large portion of tissue, LSM is chiefly adopted for imaging of low-scattering media, even if imaging in relatively opaque tissues have been demonstrated in double-sided illumination and detection arrangements (Tomer et al., 2012; Lemon et al., 2015; Ezpeleta et al., 2016). Interestingly, double-sided illumination can be potentially used with one low-NA objective to generate the light-sheet and the other opposite-sided objective chosen with a high NA to address the $3 \mathrm{D}$ photostimulation patterns. At last, it should also be considered, that the orthogonal disposition of illumination and detection objectives, ultimately limit the geometric accessibility to the sample compared to other techniques relying on a single objective.

\section{REFERENCES}

Accanto, N., Molinier, C., Tanese, D., Ronzitti, E., Newman, Z. L., Wyart, C., et al. (2018). Multiplexed temporally focused light shaping for highresolution multi-cell targeting. Optica 5, 1478-1491. doi: 10.1364/OPTICA.5. 001478

Ahrens, M. B., Orger, M. B., Robson, D. N., Li, J. M., and Keller, P. J. (2013). Wholebrain functional imaging at cellular resolution using light-sheet microscopy. Nat. Methods 10, 413-420. doi: 10.1038/nmeth.2434

Airan, R. D., Thompson, K. R., Fenno, L. E., Bernstein, H., and Deisseroth, K. (2009). Temporally precise in vivo control of intracellular signalling. Nature 458, 1025-1029. doi: 10.1038/nature07926

\section{OUTLOOK}

From this overview of the methods for $3 \mathrm{D}$ photoactivation and imaging, it is evident that these domains have tremendously advanced over the last few years. However, the combination of all-optical approaches is so far limited to use 2P scanning imaging modalities with an ETL (Dal Maschio et al., 2017; Mardinly et al., 2018; Yang et al., 2018), which is the most straightforward method for multiplane imaging, from those presented.

Nevertheless, the first steps have now been completed and we are entering an era where there will be an increasing demand for high-performance all-optical methods to tackle more complex biological questions. This will certainly prompt further developments for all-optical strategies in large excitation volumes and multi-area microscopes, where it will be possible, for instance, to activate a population of neurons in one area while monitoring the effects in another area of the brain (Lecoq et al., 2014; Chen et al., 2016). Furthermore, for imaging and photoactivation in large depths, development of microendoscopes based on miniaturized optics (Zong et al., 2017; Zou et al., 2017; Ozbay et al., 2018), able to perform all-optical manipulations, and use of three-photon excitation (Horton et al., 2013; Chen et al., 2018a; Rodríguez et al., 2018), can be envisioned.

\section{AUTHOR CONTRIBUTIONS}

EP conceived and organized the structure of the manuscript. EP and ER wrote the manuscript and prepared the figures. VE revised and contributed in writing the manuscript.

\section{FUNDING}

EP acknowledges the "Agence Nationale de la Recherche" ANR (Grants ANR-15-CE19-0001-01, 3DHoloPAc) for financial support. ER acknowledges the European Research Council SYNERGY Grant Scheme (HELMHOLTZ, ERC Grant Agreement \# 610110). VE acknowledges the National Institutes of Health (NIH) (1UF1NS107574-01), Human Frontier Science Program (HFSP) (RGP0015/2016), Fondation Bettencourt Shueller (Prix Coups d'Élan pour la Recherche Française) and AXA Research Fund.

Amir, W., Carriles, R., Hoover, E. E., Durfee, C. G., and Squier, J. A. (2007). Simultaneous imaging of multiple focal planes in scanning two-photon absorption microscope. Opt. Lett. 32, 1731-1733. doi: 10.1117/12.731868

Andrasfalvy, B. K., Zemelman, B. V., Tang, J., and Vaziri, A. (2010). Two-photon single-cell optogenetic control of neuronal activity by sculpted light. Proc. Natl. Acad. Sci. U.S.A. 107, 11981-11986. doi: 10.1073/pnas.1006620107

Anselmi, F., Ventalon, C., Begue, A., Ogden, D., Emiliani, V., Bègue, A., et al. (2011). Three-dimensional imaging and photostimulation by remote-focusing and holographic light patterning. Proc. Natl. Acad. Sci. U.S.A. 108, 1950419509. doi: $10.1073 /$ pnas. 1109111108

Bañas, A., and Glückstad, J. (2017). Holo-GPC: holographic generalized phase contrast. Opt. Commun. 392, 190-195. doi: 10.1016/j.optcom.2017.01.036 
Bañas, A., Palima, D., Villangca, M., Aabo, T., and Glückstad, J. (2014). GPC light shaper for speckle-free one- and two- photon contiguous pattern excitation. Opt. Express 7102, 5299-5310. doi: 10.1364/OE.22.005299

Becker, Y., Unger, E., Fichte, M. A. H., Gacek, D. A., Dreuw, A., Wachtveitl, J., et al. (2018). A red-shifted two-photon-only caging group for three-dimensional photorelease. Chem. Sci. 9, 2797-2802. doi: 10.1039/C7SC05182D

Bègue, A., Papagiakoumou, E., Leshem, B., Conti, R., Enke, L., Oron, D., et al. (2013). Two-photon excitation in scattering media by spatiotemporally shaped beams and their application in optogenetic stimulation. Biomed. Opt. Express 4, 2869-2879. doi: 10.1364/BOE.4.002869

Booth, M. J. (2014). Adaptive optical microscopy: the ongoing quest for a perfect image. Light Sci. Appl. 3:e165. doi: 10.1038/lsa.2014.46

Botcherby, E. J., Juskaitis, R., Booth, M. J., and Wilson, T. (2007). Aberrationfree optical refocusing in high numerical aperture microscopy. Opt. Lett. 32, 2007-2009. doi: 10.1364/OL.32.002007

Botcherby, E. J., Smith, C. W., Kohl, M. M., Débarre, D., Booth, M. J., Juskaitis, R., et al. (2012). Aberration-free three-dimensional multiphoton imaging of neuronal activity at kHz rates. Proc. Natl. Acad. Sci. U.S.A. 109, 2919-2924. doi: 10.1073/pnas.1111662109

Botcherby, E. J. J., Juškaitis, R., Wilson, T., Botcherby, E. J. J., Jus, R., Juskaitis, R., et al. (2006). Scanning two photon fluorescence microscopy with extended depth of field. Opt. Commun. 268, 253-260. doi: 10.1016/j.optcom.2006.07.026

Bovetti, S., Moretti, C., Zucca, S., Dal Maschio, M., Bonifazi, P., and Fellin, T. (2017). Simultaneous high-speed imaging and optogenetic inhibition in the intact mouse brain. Sci. Rep. 7:40041. doi: 10.1038/srep40041

Broxton, M., Grosenick, L., Yang, S., Cohen, N., Andalman, A., Deisseroth, K., et al. (2013). Wave optics theory and 3-D deconvolution for the light field microscope. Opt. Express 21, 25418-25439. doi: 10.1364/OE.21.025418

Carrillo-reid, L., Yang, W., Bando, Y., Peterka, D. S., and Yuste, R. (2016). Imprinting and recalling cortical ensembles. Science 353, 691-694. doi: 10.1126/ science.aaf7560

Carroll, E. C., Berlin, S., Levitz, J., Kienzler, M. A., Yuan, Z., Madsen, D., et al. (2015). Two-photon brightness of azobenzene photoswitches designed for glutamate receptor optogenetics. Proc. Natl. Acad. Sci. U.S.A. 112, E776-E785. doi: $10.1073 /$ pnas. 1416942112

Chaigneau, E., Ronzitti, E., Gajowa, A. M., Soler-Llavina, J. G., Tanese, D., Brureau, Y. B. A., et al. (2016). Two-photon holographic stimulation of ReaChR. Front. Cell. Neurosci. 10:234. doi: 10.3389/fncel.2016.00234

Chen, B., Huang, X., Gou, D., Zeng, J., Chen, G., Pang, M., et al. (2018a). Rapid volumetric imaging with Bessel-Beam three-photon microscopy. Biomed. Opt. Express 9, 1992-2000. doi: 10.1364/BOE.9.001992

Chen, I. W., Papagiakoumou, E., and Emiliani, V. (2018b). Towards circuit optogenetics. Curr. Opin. Neurobiol. 50, 179-189. doi: 10.1016/j.conb.2018.03.008

Chen, I.-W., Ronzitti, E., Lee, R. B., Daigle, L. T., Zeng, H., Papagiakoumou, E., et al. (2018c). Parallel holographic illumination enables sub-millisecond two-photon optogenetic activation in mouse visual cortex in vivo. bioRxiv [Preprint]. doi: $10.1101 / 250795$

Chen, J. L., Voigt, F. F., Javadzadeh, M., Krueppel, R., and Helmchen, F. (2016). Long-Range population dynamics of anatomically defined neocortical networks. eLife 5:e14679. doi: 10.7554/eLife.14679

Cheng, A., Gonçalves, J. T., Golshani, P., Arisaka, K., and Portera-Cailliau, C. (2011). Simultaneous two-photon calcium imaging at different depths with spatiotemporal multiplexing. Nat. Methods 8, 139-142. doi: 10.1038/nmeth. 1552

Cohen, N., Yang, S., Andalman, A., Broxton, M., Grosenick, L., Deisseroth, K., et al. (2014). Enhancing the performance of the light field microscope using wavefront coding. Opt. Express 22, 24817-24839. doi: 10.1364/OE.22. 024817

Cossell, L., Iacaruso, M. F., Muir, D. R., Houlton, R., Sader, E. N., Ko, H., et al. (2015). Functional organization of excitatory synaptic strength in primary visual cortex. Nature 518, 399-403. doi: 10.1038/nature14182

Dal Maschio, M., De Stasi, A. M., Benfenati, F., and Fellin, T. (2011). Threedimensional in vivo scanning microscopy with inertia-free focus control. Opt. Lett. 36, 3503-3505. doi: 10.1364/OL.36.003503

Dal Maschio, M., Difato, F., Beltramo, R., Blau, A., Benfenati, F., and Fellin, T. (2010). Simultaneous two-photon imaging and photo-stimulation with structured light illumination. Opt. Express 18, 18720-18731. doi: 10.1364/ OE.18.018720

Dal Maschio, M., Donovan, J. C., Helmbrecht, T. O., and Baier, H. (2017). Linking neurons to network function and behavior by two-photon holographic optogenetics and volumetric imaging. Neuron 94, 774-789.e5. doi: 10.1016/j. neuron.2017.04.034

Dana, H., and Shoham, S. (2012). Remotely scanned multiphoton temporal focusing by axial grism scanning. Opt. Lett. 37, 2913-2915. doi: 10.1364/OL. 37.002913

Di Leonardo, R., Ianni, F., and Ruocco, G. (2007). Computer generation of optimal holograms for optical trap arrays. Opt. Express 15, 1913-1922. doi: 10.1364/OE. 15.001913

Ducros, M., Goulam Houssen, Y., Bradley, J., de Sars, V., and Charpak, S. (2013). Encoded multisite two-photon microscopy. Proc. Natl. Acad. Sci. U.S.A. 110, 13138-13143. doi: 10.1073/pnas.1307818110

Durst, M. E., Zhu, G., and Xu, C. (2006). Simultaneous spatial and temporal focusing for axial scanning. Opt. Express 14, 12243-12254. doi: 10.1364/OE.14. 012243

Durst, M. E., Zhu, G., and Xu, C. (2008). Simultaneous spatial and temporal focusing in nonlinear microscopy. Opt. Commun. 281, 1796-1805. doi: 10.1016/ j.optcom.2007.05.071

Emiliani, V., Cohen, A. E., Deisseroth, K., and Häusser, M. (2015). All-optical interrogation of neural circuits. J. Neurosci. 35, 13917-13926. doi: 10.1523/ JNEUROSCI.2916-15.2015

Ezpeleta, E., Zurutuza, U., and Hidalgo, J. M. G. (2016). Using personality recognition techniques to improve Bayesian spam filtering. Proces. Leng. Nat. 57, 125-132. doi: 10.1038/nmeth.2064

Fahrbach, F., Voigt, F., Schmid, B., Helmchen, F., and Huisken, J. (2013). Rapid 3D light-sheet microscopy with a tunable lens. Opt. Express 21, 1963-1975. doi: 10.1364/OE.21.021010

Forli, A., Vecchia, D., Binini, N., Succol, F., Bovetti, S., Moretti, C., et al. (2018). Two-photon bidirectional control and imaging of neuronal excitability with high spatial resolution in vivo. Cell Rep. 22, 2809-2817. doi: 10.1016/j.celrep. 2018.02.063

Förster, D., Dal Maschio, M., Laurell, E., and Baier, H. (2017). An optogenetic toolbox for unbiased discovery of functionally connected cells in neural circuits. Nat. Commun. 8:116. doi: 10.1038/s41467-017-00160-z

Foust, A. J., Zampini, V., Tanese, D., Papagiakoumou, E., and Emiliani, V. (2015). Computer-generated holography enhances voltage dye fluorescence discrimination in adjacent neuronal structures. Neurophotonics 2:021007. doi: 10.1117/1.NPh.2.2.021007

Gerchberg, R. W., and Saxton, W. O. (1972). A practical algorithm for the determination of the phase from image and diffraction pictures. Optik 35, 237-246.

Glückstad, J. (1996). Phase contrast image synthesis. Opt. Commun. 130, 225-230. doi: 10.1016/0030-4018(96)00339-2

Go, M. A., Ng, P.-F., Bachor, H. A., and Daria, V. R. (2011). Optimal complex field holographic projection. Opt. Lett. 36, 3073-3075. doi: 10.1364/OL.36.003073

Go, M. A., Stricker, C., Redman, S., Bachor, H.-A. A., and Daria, V. R. (2012). Simultaneous multi-site two-photon photostimulation in three dimensions. J. Biophotonics 5, 745-753. doi: 10.1002/jbio.201100101

Göbel, W., Kampa, B. M., Helmchen, F., and Go, W. (2007). Imaging cellular network dynamics in three dimensions using fast 3D laser scanning. Nat. Methods 4, 73-79. doi: 10.1038/NMETH989

Grewe, B. F., Langer, D., Kasper, H., Kampa, B. M., and Helmchen, F. (2010). High-speed in vivo calcium imaging reveals neuronal network activity with near-millisecond precision. Nat. Methods 7, 399-405. doi: 10.1038/nmeth.1453

Grewe, B. F., Voigt, F. F., van 't Hoff, M., Helmchen, F., van 't Hoff, M., Helmchen, F., et al. (2011). Fast two-layer two-photon imaging of neuronal cell populations using an electrically tunable lens. Biomed. Opt. Express 2, 2035-2046. doi: 10.1364/BOE.2.002035

Grosenick, L. M., Broxton, M., Kim, C. K., Liston, C., Poole, B., Yang, S., et al. (2017). Identification of cellular-activity dynamics across large tissue volumes in the mammalian brain. bioRxiv [Preprint]. doi: 10.1101/132688

Guruge, C., Ouedraogo, Y. P., Comitz, R. L., Ma, J., Losonczy, A., and Nesnas, N. (2018). Improved synthesis of caged glutamate and caging each functional group. ACS Chem. Neurosci. 9, 2713-2727. doi: 10.1021/acschemneuro.8b00152 
Haist, T., Schönleber, M., and Tiziani, H. (1997). Computer-generated holograms from 3D-objects written on twisted-nematic liquid crystal displays. Opt. Commun. 140, 299-308. doi: 10.1016/S0030-4018(97)00192-2

Herlitze, S., and Landmesser, L. T. (2007). New optical tools for controlling neuronal activity. Curr. Opin. Neurobiol. 17, 87-94. doi: 10.1016/j.conb.2006. 12.002

Hernandez, O., Papagiakoumou, E., Tanese, D., Fidelin, K., Wyart, C., and Emiliani, V. (2016). Three-dimensional spatiotemporal focusing of holographic patterns. Nat. Commun. 7:11928. doi: 10.1038/ncomms11928

Horton, N. G., Wang, K., Kobat, D., Clark, C. G., Wise, F. W., Schaffer, C. B., et al. (2013). In vivo three-photon microscopy of subcortical structures within an intact mouse brain. Nat. Photonics 7, 205-209. doi: 10.1038/nphoton.2012.336

Isobe, K., Hashimoto, H., Suda, A., Kannari, F., Kawano, H., Mizuno, H., et al. (2010). Measurement of two-photon excitation spectrum used to photoconvert a fluorescent protein (Kaede) by nonlinear Fourier-transform spectroscopy. Biomed. Opt. Express 1, 687-693. doi: 10.1364/BOE.1.000687

Ji, N., Freeman, J., and Smith, S. L. (2016). Technologies for imaging neural activity in large volumes. Nat. Neurosci. 19, 1154-1164. doi: 10.1038/nn.4358

Ji, N., Magee, J. C., and Betzig, E. (2008). High-speed, low-photodamage nonlinear imaging using passive pulse splitters. Nat. Methods 5, 197-202. doi: 10.1038/ nmeth.1175

Katona, G., Kaszás, A., Turi, G. F., Hájos, N., Tamás, G., Vizi, E. S., et al. (2011). Roller Coaster Scanning reveals spontaneous triggering of dendritic spikes in CA1 interneurons. Proc. Natl. Acad. Sci. U.S.A. 108, 2148-2153. doi: 10.1073/ pnas. 1009270108

Katona, G., Szalay, G., Maák, P., Kaszás, A., Veress, M., Hillier, D., et al. (2012). Fast two-photon in vivo imaging with three-dimensional random-access scanning in large tissue volumes. Nat. Methods 9, 201-208. doi: 10.1038/nMeth.1851

Keller, P. J., and Ahrens, M. B. (2015). Visualizing whole-brain activity and development at the single-cell level using light-sheet microscopy. Neuron 85 , 462-483. doi: 10.1016/j.neuron.2014.12.039

Kim, E. H., Chin, G., Rong, G., Poskanzer, K. E., and Clark, H. A. (2018). Optical probes for neurobiological sensing and imaging. Acc. Chem. Res. 51, 1023-1032. doi: 10.1021 /acs.accounts.7b00564

Kirkby, P. A., Srinivas Nadella, K. M., and Silver, R. A. (2010). A compact Acoustooptic lens for 2D and 3D femtosecond based 2-photon microscopy. Opt. Express 18, 13721-13745. doi: 10.1364/OE.18.013720

Klapoetke, N. C., Murata, Y., Kim, S. S., Pulver, S. R., Birdsey-Benson, A., Cho, Y. K., et al. (2014). Independent optical excitation of distinct neural populations. Nat. Methods 11, 338-346. doi: 10.1038/nmeth.2836

Kong, L., Tang, J., Little, J. P., Yu, Y., Lämmermann, T., Lin, C. P., et al. (2015). Continuous volumetric imaging via an optical phase-locked ultrasound lens. Nat. Methods 12, 759-762. doi: 10.1038/nmeth.3476

König, K., Becker, T. W., Fischer, P., Riemann, I., and Halbhuber, K.-J. (1999). Pulse-length dependence of cellular response to intense near-infrared laser pulses in multiphoton microscopes. Opt. Lett. 24, 113-115. doi: 10.1364/OL. 24.000113

Kwon, T., Sakamoto, M., Peterka, D. S., and Yuste, R. (2017). Attenuation of synaptic potentials in dendritic spines. Cell Rep. 20, 1100-1110. doi: 10.1016/ j.celrep.2017.07.012

Lecoq, J., Savall, J., Vuèiniæ, D., Grewe, B. F., Kim, H., Li, J. Z., et al. (2014). Visualizing mammalian brain area interactions by dual-axis two-photon calcium imaging. Nat. Neurosci. 17, 1825-1829. doi: 10.1038/nn.3867

Lemon, W. C., Pulver, S. R., Höckendorf, B., McDole, K., Branson, K., Freeman, J., et al. (2015). Whole-central nervous system functional imaging in larval Drosophila. Nat. Commun. 6:7924. doi: 10.1038/ncomms8924

Leshem, B., Hernandez, O., Papagiakoumou, E., Emiliani, V., and Oron, D. (2014). When can temporally focused excitation be axially shifted by dispersion? Opt. Express 22, 7087-7098. doi: 10.1364/OE.22.007087

Levitz, J., Pantoja, C., Gaub, B., Janovjak, H., Reiner, A., Hoagland, A., et al. (2013). Optical control of metabotropic glutamate receptors. Nat. Neurosci. 16, 507-516. doi: 10.1038/nn.3346

Liesener, J., Reicherter, M., Haist, T., and Tiziani, H. J. (2000). Multi-functional optical tweezers using computer-generated holograms. Opt. Commun. 185, 77-82. doi: 10.1016/S0030-4018(00)00990-1

Lim, D., Chu, K. K., and Mertz, J. (2008). Wide-field fluorescence sectioning with hybrid speckle and uniform-illumination microscopy. Opt. Lett. 33, 1819-1821. doi: $10.1364 /$ OL.33.001819
Lu, R., Sun, W., Liang, Y., Kerlin, A., Bierfeld, J., Seelig, J. D., et al. (2017). Videorate volumetric functional imaging of the brain at synaptic resolution. Nat. Neurosci. 20, 620-628. doi: 10.1038/nn.4516

Lutz, C., Otis, T. S., DeSars, V., Charpak, S., Digregorio, D. A., and Emiliani, V. (2008). Holographic photolysis of caged neurotransmitters. Nat. Methods 5, 821-827. doi: $10.1038 /$ nmeth.1241

Mardinly, A. R., Oldenburg, I. A., Pégard, N. C., Sridharan, S., Lyall, E. H., Chesnov, K., et al. (2018). Precise multimodal optical control of neural ensemble activity. Nat. Neurosci. 21, 881-893. doi: 10.1038/s41593-018-0139-8

Mermillod-Blondin, A., McLeod, E., and Arnold, C. B. (2008). High-speed varifocal imaging with a tunable acoustic gradient index of refraction lens. Opt. Lett. 33, 2146-2148. doi: 10.1364/OL.33.002146

Nadella, K. M. N. S., Roš, H., Baragli, C., Griffiths, V. A., Konstantinou, G., Koimtzis, T., et al. (2016). Random access scanning microscopy for 3D imaging in awake behaving animals. Nat. Methods 13, 1001-1004. doi: 10.1038/nMeth. 4033

Nikolenko, V., Poskanzer, K. E., and Yuste, R. (2007). Two-photon photostimulation and imaging of neural circuits. Nat. Methods 4, 943-950. doi: 10.1038/NMETH1105

Nikolenko, V., Watson, B. O., Araya, R., Woodruff, A., Peterka, D. S., and Yuste, R. (2008). SLM microscopy: scanless two-photon imaging and photostimulation with spatial light modulators. Front. Neural Circuits 2:5. doi: 10.3389/neuro.04. 005.2008

Olarte, O. E., Andilla, J., Artigas, D., and Loza-Alvarez, P. (2015). Decoupled illumination detection in light sheet microscopy for fast volumetric imaging. Optica 2, 702-705. doi: 10.1364/OPTICA.2.000702

Olivié, G., Giguère, D., Vidal, F., Ozaki, T., Kieffer, J.-C., Nada, O., et al. (2008). Wavelength dependence of femtosecond laser ablation threshold of corneal stroma. Opt. Express 16, 4121-4129. doi: 10.1364/OE.16. 004121

Oron, D., Papagiakoumou, E., Anselmi, F., and Emiliani, V. (2012). Two-photon optogenetics. Prog. Brain Res. 196, 119-143. doi: 10.1016/B978-0-444-59426-6. 00007-0

Oron, D., Tal, E., and Silberberg, Y. (2005). Scanningless depth-resolved microscopy. Opt. Express 13, 1468-1476. doi: 10.1364/OPEX.13.001468

Ozbay, B. N., Futia, G. L., Ma, M., Bright, V. M., Gopinath, J. T., Hughes, E. G., et al. (2018). Three dimensional two-photon imaging of neuronal activity in freely moving mice using a miniature fiber coupled microscope with active axial-scanning. bioRxiv [Preprint]. doi: 10.1101/226431

Packer, A. M., Peterka, D. S., Hirtz, J. J., Prakash, R., Deisseroth, K., Yuste, R., et al. (2012). Two-photon optogenetics of dendritic spines and neural circuits. Nat. Methods 9, 1171-1179. doi: 10.1038/nmeth.2249

Packer, A. M., Russell, L. E., Dalgleish, H. W. P., and Häusser, M. (2015). Simultaneous all-optical manipulation and recording of neural circuit activity with cellular resolution in vivo. Nat. Methods 12, 140-146. doi: 10.1038/nmeth. 3217

Papagiakoumou, E. (2013). Optical developments for optogenetics. Biol. Cell 105, 443-464. doi: 10.1111/boc.201200087

Papagiakoumou, E., Anselmi, F., Bègue, A., de Sars, V., Glückstad, J., Isacoff, E. Y., et al. (2010). Scanless two-photon excitation of channelrhodopsin-2. Nat. Methods 7, 848-854. doi: 10.1038/nmeth.1505

Papagiakoumou, E., Bègue, A., Leshem, B., Schwartz, O., Stell, B. M., Bradley, J., et al. (2013). Functional patterned multiphoton excitation deep inside scattering tissue. Nat. Photonics 7, 274-278. doi: 10.1038/nphoton.2013.9

Papagiakoumou, E., de Sars, V., Oron, D., and Emiliani, V. (2008). Patterned two-photon illumination by spatiotemporal shaping of ultrashort pulses. Opt. Express 16, 22039-22047. doi: 10.1364/OE.16.022039

Papagiakoumou, E., Ronzitti, E., Chen, I.-W., Gajowa, M., Picot, A., and Emiliani, V. (2018). Two-photon optogenetics by computer-generated holography. Neuromethods 133, 175-197. doi: 10.1007/978-1-49397417-7_10

Pégard, N., Mardinly, A., Oldenburg, I., Waller, L., and Adesnik, H. (2018). Partially Coherent Holographic temporal focusing for $3 D$ light sculpting with single neuron resolution. Opt. InfoBase Conf. Pap. Part F88-B, 4-5. doi: 10.1364/ BRAIN.2018.BW2C. $2 \$ \wedge\{*\} \$$

Pégard, N. M., Oldenburg, I., Sridharan, S., Walller, L., and Adesnik, H. (2017). 3D scanless holographic optogenetics with temporal focusing. Nat. Commun. 8:1228. doi: 10.1038/s41467-017-01031-3 
Peron, S. P., Freeman, J., Iyer, V., Guo, C., and Svoboda, K. (2015). A cellular resolution map of barrel cortex activity during tactile behavior. Neuron 86, 783-799. doi: 10.1016/j.neuron.2015.03.027

Picot, A., Dominguez, S., Liu, C., Chen, I. W., Tanese, D., Ronzitti, E., et al. (2018). Temperature rise under two-photon optogenetic brain stimulation. Cell Rep. 24, 1243-1253.e5. doi: 10.1016/j.celrep.2018.06.119

Piestun, R., Spektor, B., and Shamir, J. (1996). Wave fields in three dimensions: analysis and synthesis. J. Opt. Soc. Am. A 13, 1837-1848. doi: 10.1364/JOSAA. 13.001837

Power, R. M., and Huisken, J. (2017). A guide to light-sheet fluorescence microscopy for multiscale imaging. Nat. Methods 14, 360-373. doi: 10.1038/ nmeth.4224

Prakash, R., Yizhar, O., Grewe, B., Ramakrishnan, C., Wang, N., Goshen, I., et al. (2012). Two-photon optogenetic toolbox for fast inhibition, excitation and bistable modulation. Nat. Methods 9, 1171-1179. doi: 10.1038/nmeth.2215

Prevedel, R., Verhoef, A. J., Pernía-Andrade, A. J., Weisenburger, S., Huang, B. S., Nöbauer, T., et al. (2016). Fast volumetric calcium imaging across multiple cortical layers using sculpted light. Nat. Methods 13, 1021-1028. doi: 10.1038/ nmeth.4040

Prevedel, R., Yoon, Y.-G., Hoffmann, M., Pak, N., Wetzstein, G., Kato, S., et al. (2014). Simultaneous whole-animal 3D imaging of neuronal activity using light-field microscopy. Nat. Methods 11, 727-730. doi: 10.1038/nmeth.2964

Quirin, S., Peterka, D. S., and Yuste, R. (2013). Instantaneous three-dimensional sensing using spatial light modulator illumination with extended depth of field imaging. Opt. Express 21, 16007-16021. doi: 10.1364/OE.21.016007

Quirin, S., Vladimirov, N., Yang, C.-T., Peterka, D. S., Yuste, R., and Ahrens, M. B. (2016). Calcium imaging of neural circuits with extended depth-of-field light-sheet microscopy. Opt. Lett. 41, 855-858. doi: 10.1364/OL.41.000855

Reddy, G. D., Kelleher, K., Fink, R., and Saggau, P. (2008). Three-dimensional random access multiphoton microscopy for functional imaging of neuronal activity. Nat. Neurosci. 11, 713-720. doi: 10.1038/nn.2116

Rickgauer, J. P., Deisseroth, K., and Tank, D. W. (2014). Simultaneous cellularresolution optical perturbation and imaging of place cell firing fields. Nat. Neurosci. 17, 1816-1824. doi: 10.1038/nn.3866

Rickgauer, J. P., and Tank, D. W. (2009). Two-photon excitation of channelrhodopsin-2 at saturation. Proc. Natl. Acad. Sci. U.S.A. 106, 15025-15030. doi: 10.1073/pnas.0907084106

Rodríguez, C., Liang, Y., Lu, R., and Ji, N. (2018). Three-photon fluorescence microscopy with an axially elongated Bessel focus. Opt. Lett. 43, 1914-1917. doi: 10.1364/OL.43.001914

Ronzitti, E., Conti, R., Zampini, V., Tanese, D., Foust, A. J., Klapoetke, N., et al. (2017a). Sub-millisecond optogenetic control of neuronal firing with twophoton holographic photoactivation of Chronos. J. Neurosci. 37, 10679-10689. doi: 10.1523/JNEUROSCI.1246-17.2017

Ronzitti, E., Ventalon, C., Canepari, M., Forget, B. C., Papagiakoumou, E., and Emiliani, V. (2017b). Recent advances in patterned photostimulation for optogenetics. J. Opt. 19:113001. doi: 10.1088/2040-8986/aa8299

Rupprecht, P., Prendergast, A., Wyart, C., and Friedrich, R. W. (2016). Remote $\mathrm{z}$-scanning with a macroscopic voice coil motor for fast $3 \mathrm{D}$ multiphoton laser scanning microscopy. Biomed. Opt. Express 7, 1656-1671. doi: 10.1364/BOE.7. 001656

Seelig, J. D., and Jayaraman, V. (2015). Neural dynamics for landmark orientation and angular path integration. Nature 521, 186-191. doi: 10.1038/nature14446

Shemesh, O. A., Tanese, D., Zampini, V., Linghu, C., Piatkevich, K., Ronzitti, E., et al. (2017). Temporally precise single-cell resolution optogenetics. Nat. Neurosci. 20, 1796-1806. doi: 10.1038/s41593-0170018-8

Sofroniew, N. J., Flickinger, D., King, J., and Svoboda, K. (2016). A large field of view two-photon mesoscope with subcellular resolution for in vivo imaging. eLife 5:e14472. doi: 10.7554/eLife.14472
Stirman, J. N., Smith, I. T., Kudenov, M. W., and Smith, S. L. (2016). Wide field-ofview, multi-region, two-photon imaging of neuronal activity in the mammalian brain. Nat. Biotechnol. 34, 857-862. doi: 10.1038/nbt.3594

Sun, B., Salter, P. S., Roider, C., Strauss, J., Heberle, J., and Booth, M. J. (2018). Four-dimensional light shaping?: manipulating ultrafast spatiotemporal foci in space and time. Light Sci. Appl. 7:17117. doi: 10.1038/lsa. 2017.117

Szabo, V., Ventalon, C., De Sars, V., Bradley, J., and Emiliani, V. (2014). Spatially selective holographic photoactivation and functional fluorescence imaging in freely behaving mice with a fiberscope. Neuron $84,1157-1169$. doi: 10.1016/j. neuron.2014.11.005

Tanese, D., Weng, J.-Y., Zampini, V., De Sars, V., Canepari, M., Rozsa, B., et al. (2017). Imaging membrane potential changes from dendritic spines using computer-generated holography. Neurophotonics 4:031211. doi: 10.1117/ 1.NPh.4.3.031211

Tomer, R., Khairy, K., Amat, F., and Keller, P. J. (2012). Quantitative high-speed imaging of entire developing embryos with simultaneous multiview light-sheet microscopy. Nat. Methods 9, 755-763. doi: 10.1038/nmeth.2062

Vogt, N. (2015). All-optical electrophysiology in behaving animals. Nat. Methods 12:101. doi: 10.1038/nmeth.3272

Yang, S., Papagiakoumou, E., Guillon, M., de Sars, V., Tang, C. M., and Emiliani, V. (2011). Three-dimensional holographic photostimulation of the dendritic arbor. J. Neural Eng. 8:46002. doi: 10.1088/1741-2560/8/4/046002

Yang, W., Carrillo-reid, L., Bando, Y., Peterka, D. S., and Yuste, R. (2018). Simultaneous two-photon optogenetics and imaging of cortical circuits in three dimensions. eLife 7:e32671. doi: 10.7554/eLife.32671

Yang, W., Miller, J. K., Carrillo-Reid, L., Pnevmatikakis, E., Paninski, L., Yuste, R., et al. (2016). Simultaneous multi-plane imaging of neural circuits. Neuron 89 , 269-284. doi: 10.1016/j.neuron.2015.12.012

Yang, W., and Yuste, R. (2017). In vivo imaging of neural activity. Nat. Methods 14, 349-359. doi: 10.1038/nmeth.4230

Yang, W., and Yuste, R. (2018). Holographic imaging and photostimulation of neural activity. Curr. Opin. Neurobiol. 50, 211-221. doi: 10.1016/j.conb.2018. 03.006

Yizhar, O., Fenno, L. E., Davidson, T. J., Mogri, M., and Deisseroth, K. (2011). Optogenetics in neural systems. Neuron 71, 9-34. doi: 10.1016/j.neuron.2011. 06.004

Zhu, G., van Howe, J., Durst, M., Zipfel, W., and Xu, C. (2005). Simultaneous spatial and temporal focusing of femtosecond pulses. Opt. Express 13, 21532159. doi: 10.1364/OPEX.13.002153

Zong, W., Wu, R., Li, M., Hu, Y., Li, Y., Li, J., et al. (2017). Fast high-resolution miniature two-photon microscopy for brain imaging in freely behaving mice. Nat. Methods 14, 713-719. doi: 10.1038/nmeth.4305

Zou, Y., Chau, F. S., and Zhou, G. (2017). Ultra-compact optical zoom endoscope using solid tunable lenses. Opt. Express 25, 20675-20688. doi: 10.1364/OE.25. 020675

Conflict of Interest Statement: The authors declare that the research was conducted in the absence of any commercial or financial relationships that could be construed as a potential conflict of interest.

The handling Editor declared a past co-authorship with the authors.

Copyright $\odot 2018$ Ronzitti, Emiliani and Papagiakoumou. This is an open-access article distributed under the terms of the Creative Commons Attribution License (CC BY). The use, distribution or reproduction in other forums is permitted, provided the original author(s) and the copyright owner(s) are credited and that the original publication in this journal is cited, in accordance with accepted academic practice. No use, distribution or reproduction is permitted which does not comply with these terms. 